01

\title{
Сдвиги частоты магнитного резонанса в тандемном Cs-K-магнитометре, обусловленные спиновым обменом
}

\author{
(C) В.А. Картошкин \\ ФТИ им. А.Ф. Иофрфе РАН, \\ 194021 Санкт-Петербург, Россия \\ e-mail: victor.kart@mail.ioffe.ru
}

Поступила в редакцию 31.03.2020 г.

В окончательной редакции 31.03.2020 г.

Принята к публикации 04.05.2020 г.

Рассчитаны сдвиги частоты магнитного резонанса атомов Cs и K, обусловленные спиновым обменом в условиях оптической ориентации атомов. Изучаемая ситуация характерна для случая тандемного магнитометра на смеси атомов цезия и калия, находящихся в одной рабочей камере.

Ключевые слова: спиновый обмен, поперечные сечения, сдвиги частоты магнитного резонанса.

DOI: $10.21883 /$ OS.2020.09.49859.125-20

\section{Введение}

В настоящее время большую роль в проведении разного рода магнитных измерений играют квантовые магнитометры с оптической накачкой (КМOH) [1,2]. В качестве рабочих сред такого рода магнитометров выступают атомы щелочных металлов в основном состоянии [3], смеси щелочных атомов и возбужденных атомов гелия в метастабильном состоянии $2^{3} S_{1}$ [4], возбужденные атомы гелия в метастабильном состоянии $2^{3} S_{1}[5]$.

Возросшее внимание к КМОН обусловлено, в частности, использование квантовых магнитометров в прикладных целях в качестве элементов, входящих, в частности, в квантовые гироскопы и магнитоэнцефалографы. Использование в качестве рабочих сред в этих устройствах атомов щелочных металлов вместе с тем приводит к определенным проблемам. Эти проблемы обусловлены, в первую очередь, столкновениями с участием щелочных атомов. Подобного рода столкновения сопровождаются процессом спинового обмена, т.е. процессом обмена электронной поляризацией между сталкивающимися частицами, если последние обладают не скомпенсированным электронным спином. В дальнейшем за время между столкновениями происходит перераспределение электронной поляризации между электронной и ядерной степенями свободы щелочного атома, обусловленное сверхтонким взаимодействием. Как и практически любой процесс, спиновой обмен характеризуется как положительными моментами - возможностью передачи поляризации партнеру столкновения от предварительно оптически ориентированных атомов, так и отрицательными. К последним можно отнести уширение линий магнитного резонанса атомов, что отрицательно сказывается на точностных характеристиках $\mathrm{KMOH}$, а также возникновение сдвигов частоты магнитного резонанса сталкивающихся атомов. Следует отметить, что в слу- чае, когда спиновый обмен сопровождается неупругим процессом - хемоионизацией, как это имеет место, например, в [4], последний процесс существенно влияет как на сечения спинового обмена [6], так и на сдвиги частоты магнитного резонанса [7].

Для реализации определенного круга метрологических задач требуется сочетание быстродействия прибора и точности измерения. Зачастую подобные свойства оказывается трудно реализовать в одном устройстве, и тогда для решения поставленных задач используются квантовые магнитометры, построенные по типу „тандема“. Это устройство, которое в рабочей камере содержит смесь атомов щелочных металлов.

В [8] была предложена схема магнитометра, в рабочей камере которого находилась смесь щелочных атомов Cs и K. В устройстве поляризованные атомы Cs использовались для создания $\mathrm{M}_{\mathrm{x}}$-магнитометра, а поляризованные атомы $\mathrm{K}$ для создания $\mathrm{M}_{\mathrm{z}}$-магнитометра. Оба щелочных атома оптически ориентировались резонансным циркулярно поляризованным светом газоразрядных ламп. Для уменьшения деполяризации атомов при столкновении со стенкой камеры поглощения, в которой находилась смесь щелочных атомов, она покрывалась антирелаксационным покрытием - парафином. Таким образом, основным релаксационным процессом в камере поглощения были столкновения поляризованных щелочных атомов между собой, т.е. спиновый обмен. Как отмечалось выше, этот процесс приводит, в частности, к сдвигу частоты линий магнитного резонанса сталкивающихся атомов.

Присутствие в камере поглощения смеси щелочных атомов ведет к тому, что в условиях оптической ориентации в камере поглощения происходят столкновения как атомов К в основном состоянии между собой (то же имеет место для атомов Cs в основном состоянии), так и столкновения между атомами K и Cs. B работе будет рассмотрено влияние спин-обменных столкнове- 
ний щелочных атомов цезия и калия на сдвиги частоты магнитного резонанса этих атомов.

\section{1. Спин-обменные сдвиги частоты для смеси щелочных атомов}

Рассмотрение спин-обменных сдвигов частоты магнитного резонанса в смеси щелочных атомов было проведено в [9]. Эволюция матрицы плотности атомов А описывается следующим уравнением:

$$
\begin{aligned}
\frac{d^{(A)}}{d t} \rho= & {\left[\left(\frac{\partial}{\partial t}\right)_{\mathrm{AB}}+\left(\frac{\partial}{\partial t}\right)_{\mathrm{AA}}+\left(\frac{\partial}{\partial t}\right)_{\mathrm{AC}}\right.} \\
& \left.+\left(\frac{\partial}{\partial t}\right)_{W}+\left(\frac{\partial}{\partial t}\right)_{H}\right]^{(A)} \rho .
\end{aligned}
$$

Здесь первое слагаемое описывает изменение матрицы плотности из-за столкновений атомов А и В, второе слагаемое - изменение матрицы плотности из-за столкновений атомов А и А, третье слагаемое - изменение матрицы плотности из-за столкновений атомов А с атомами буферного газа $\mathrm{C}$, четвертое слагаемое - влияние диффузии к стенкам камеры поглощения, последнее слагаемое - влияние взаимодействия атомов с постоянным магнитным полем. Поскольку в [8] эксперименты проводились в камере поглощения с антирелаксационным покрытием без буферного газа, то третьим и четвертым слагаемыми можно пренебречь.

В соответствии с [9] учет только спин-обменных столкновений приводит к следующим выражениям для сдвигов частоты магнитного резонанса двух сверхтонких состояний щелочного атома:

$$
\Delta \omega( \pm)=\delta^{(1)} \omega( \pm)+\delta^{(2)} \omega .
$$

Здесь первое слагаемое обусловлено возникновением у атома А добавки к поперечной компоненте ориентации в столкновениях с продольно ориентированными атомами В, а второй член обусловлен переносом поперечной ориентации с одного подуровня $F$ атома А на другой в результате столкновений. Представленные в (2) сдвиги в соответствии с [9] имеют следующий вид:

$$
\begin{gathered}
\delta^{(1)} \omega(+)=-\frac{P_{z}(B)}{2\left(2 I_{\mathrm{A}}+1\right)}\left[\overline{\bar{\gamma}}_{\mathrm{AB}}-\overline{\bar{\gamma}}_{\mathrm{AA}} B_{-}\left(\frac{2 I_{\mathrm{A}}-1}{2 I_{\mathrm{A}}+1}\right)^{1 / 2}\right], \\
\delta^{(1)} \omega(-)=-\frac{P_{z}(B)}{2\left(2 I_{\mathrm{A}}+1\right)}\left[\overline{\bar{\gamma}}_{\mathrm{AB}}+\overline{\bar{\gamma}}_{\mathrm{AA}} B_{+}\left(\frac{2 I_{\mathrm{A}}+3}{2 I_{\mathrm{A}}+1}\right)^{1 / 2}\right], \\
\delta^{(2)} \omega=-\frac{C}{\bar{\omega}_{0}}\left\{\left(2 \bar{\gamma}_{\mathrm{AA}}+3 \bar{\gamma}_{\mathrm{AB}}\right)^{2}-\left[\overline{\bar{\gamma}}_{\mathrm{AA}} P_{s}(B)\right]^{2}\right\} .
\end{gathered}
$$

Здесь $\delta^{(1)} \omega(+)$ - сдвиг частоты магнитного резонанса для сверхтонкого состояния $\mathbf{F}=\mathbf{S}+\mathbf{I}$ (где $\mathbf{S}-$ электронный спин, в случае щелочных атомов он равен $1 / 2, \mathbf{I}-$ ядерный спин щелочного атома), $\delta^{(1)} \omega(-)-$ сдвиг частоты магнитного резонанса для сверхтонкого состояния $\mathbf{F}=\mathbf{S}-\mathbf{I}, P_{z}(B)-$ поляризация частицы В, $\mathbf{I}_{\mathrm{A}}$ - ядерный спин частицы $\mathrm{A}, \overline{\bar{\gamma}}_{\mathrm{AB}}$ и $\overline{\bar{\gamma}}_{\mathrm{AA}}-$ мнимые части комплексной скорости спинового обмена $\gamma$, которая может быть выражена через комплексное сечение спинового обмена. Мнимые части комплексной скорости спинового обмена можно представить в виде $\overline{\bar{\gamma}}_{\mathrm{AB}}=\left\langle v_{\mathrm{AB}}\right\rangle N_{\mathrm{B}} \overline{\bar{q}}_{\mathrm{AB}}$, где $N_{\mathrm{B}}-$ концентрация частиц сорта $\mathrm{B},\left\langle v_{\mathrm{AB}}\right\rangle=\left(8 k_{B} T / \pi \mu_{\mathrm{AB}}\right)^{1 / 2}-$ средняя относительная тепловая скорость сталкивающихся частиц (здесь $k_{B}-$ постоянная Больцмана, $T-$ температура, $\mu_{\mathrm{AB}}-$ приведенная масса), $\overline{\bar{q}}_{\mathrm{AB}}-$ мнимая часть сечения спинового обмена сталкивающихся частиц, $\bar{\omega}_{0}=H_{0}\left|g_{s}\right| \mu_{B} / \hbar$ (частота электронной прецессии), $H_{0}$ - постоянное магнитное поле, $g_{s}-g$-фактор электрона, $\mu_{B}$ - магнетон Бора. Индексы АА и АВ соответствуют столкновениям между одинаковыми или разными щелочными атомами соответственно, величины $C, B_{ \pm}$в соответствии с [9] имеют вид

$$
\begin{gathered}
B_{+}=\frac{2 I_{\mathrm{A}}+2}{6}\left(\frac{2 I_{\mathrm{A}}+3}{2 I_{\mathrm{A}}+1}\right)^{1 / 2}, \\
B_{-}=\frac{2 I_{\mathrm{A}}}{6}\left(\frac{2 I_{\mathrm{A}}-1}{2 I_{\mathrm{A}}+1}\right)^{1 / 2}, \\
C=\frac{2 I_{\mathrm{A}}\left(2 I_{\mathrm{A}}+2\right)\left(2 I_{\mathrm{A}}+3\right)\left(2 I_{\mathrm{A}}-1\right)}{288\left(2 I_{\mathrm{A}}+1\right)^{4}} .
\end{gathered}
$$

Таким образом, для расчета интересующих нас сдвигов частоты магнитного резонанса, обусловленных спиновым обменом, в соответствии с (3)-(5) необходимо знать величины комплексных сечений спинового обмена (в первую очередь их мнимых частей) для следующих пар сталкивающихся атомов: $\mathrm{Cs}-\mathrm{Cs}, \mathrm{Cs}-\mathrm{K}$ и $\mathrm{K}-\mathrm{K}$.

\section{2. Комплексные сечения спинового обмена атомов Cs-Cs, Cs-K и K-K}

Столкновения щелочных атомов можно описать с помощью соответствующих потенциалов взаимодействия. Поскольку в основном состоянии все щелочные атомы имеют нескомпенсированный электронный спин $(\mathbf{S}=\mathbf{1} / \mathbf{2})$, то образующийся в процессе столкновения димер может быть описан с помощью двух потенциалов, соответствующих полным электронным спинам $\mathbf{S}=\mathbf{0}$ (синглетный терм $X^{1} \Sigma^{+}$) и $\mathbf{S}=\mathbf{1}$ (триплетный терм $\left.a^{3} \Sigma^{+}\right)$. В работах [10-12] на основании потенциалов взаимодействия между щелочными атомами в основном состоянии были рассчитаны комплексные сечения спинового обмена для пар сталкивающихся атомов $\mathrm{Cs}-\mathrm{Cs}$, $\mathrm{Cs}-\mathrm{K}$ и $\mathrm{K}-\mathrm{K}$ :

$$
q=\bar{q}+i \overline{\bar{q}} .
$$

Здесь действительная часть комплексного сечения $(\bar{q})$ определяет передачу поляризации в столкновении, релаксацию и образование высших поляризационных моментов [13], в то время как мнимая часть сечения $(\overline{\bar{q}})$ 
Зависимость коэффициентов, входящих в (3)-(7), от ядерного спина $I_{\mathrm{A}}$

\begin{tabular}{c|c|c}
\hline Коэффициент & $I_{\mathbf{A}}(\mathrm{Cs})=7 / 2$ & $I_{\mathbf{A}}(\mathrm{K})=3 / 2$ \\
\hline$B_{+}$ & $\frac{3}{2} \sqrt{\frac{5}{4}}$ & $\frac{5}{6} \sqrt{\frac{3}{2}}$ \\
\hline$B_{-}$ & $\frac{7}{6} \sqrt{\frac{3}{4}}$ & $\frac{1}{2} \sqrt{\frac{1}{2}}$ \\
\hline$\left(\frac{2 I_{\mathrm{A}}-1}{2 I_{\mathrm{A}}+1}\right)^{1 / 2}$ & $\sqrt{\frac{3}{4}}$ & $\sqrt{\frac{1}{2}}$ \\
\hline$\left(\frac{2 I_{\mathrm{A}}+3}{2 I_{\mathrm{A}}+1}\right)^{1 / 2}$ & $\sqrt{\frac{5}{4}}$ & $\sqrt{\frac{3}{2}}$ \\
\hline$C$ & 0.0032 & 0.0012
\end{tabular}

определяет сдвиг частоты магнитного резонанса в системе зеемановских и сверхтонких уровней атомов [14].

\section{Система Cs-Cs}

Комплексные сечения спинового обмена для атомов были рассчитаны в работе [10] на основании потенциалов взаимодействия, представленных в $[15,16]$. Атом цезия $\left({ }^{133} \mathrm{Cs}\right)$ в основном состоянии $6 s 5 p^{6}$ обладает электронным спином $S=\mathbf{1} / \mathbf{2}$ и ядерным спином $I=\mathbf{7} / \mathbf{2}$. Таким образом, у атома цезия существует два сверхтонких уровня в основном состоянии: $\mathbf{F}=\mathbf{3}$ и $\mathbf{F}=\mathbf{4}$.

\section{Система Cs-K}

В [11] были рассчитаны комплексные сечения спинового обмена между атомами цезия и калия. При расчете были использованы потенциалы взаимодействия, полученные в $[17,18]$. В природе существуют два изотопа калия - ${ }^{39} \mathrm{~K}$ и ${ }^{41} \mathrm{~K}$. В тандеме $\mathrm{Cs}-\mathrm{K}$ использовались атомы изотопа ${ }^{39} \mathrm{~K}$ в основном состоянии $4 s 3 p^{6}$, обладающие ядерным спином $\mathbf{I}=\mathbf{3} / \mathbf{2}$ и имеющие два сверхтонких состояния $\mathbf{F}=\mathbf{2}$ и $\mathbf{F}=\mathbf{1}$, и атомы ${ }^{133} \mathrm{Cs}$ в основном состоянии $6 s 5 p^{6}$, обладающие электронным спином $S=\mathbf{1} / \mathbf{2}$ и ядерным спином $I=7 / 2$.

\section{Система K-K}

В [12] были рассчитаны комплексные сечения спинового обмена для пары атомов ${ }^{39} \mathrm{~K}-{ }^{39} \mathrm{~K}$ на основании потенциалов взаимодействия из работ [19-21]. Атомы изотопа калия ${ }^{39} \mathrm{~K}$ в основном $4 s 3 p^{6}$ обладают электронным спином $S=1 / 2$ и ядерным спином $I=3 / 2$.

На рис. 1 и 2 представлены усредненные по скоростям действительные и мнимые части комплексного сечения спинового обмена рассматриваемых пар атомов, рассчитанные в [10-12]. Поскольку в выражения для сдвигов частоты (3)-(5), обусловленных спиновым обменом, входят мнимые части ( $\overline{\bar{\gamma}}_{\mathrm{AB}}$ и $\left.\overline{\bar{\gamma}}_{\mathrm{AA}}\right)$ комплексной скорости

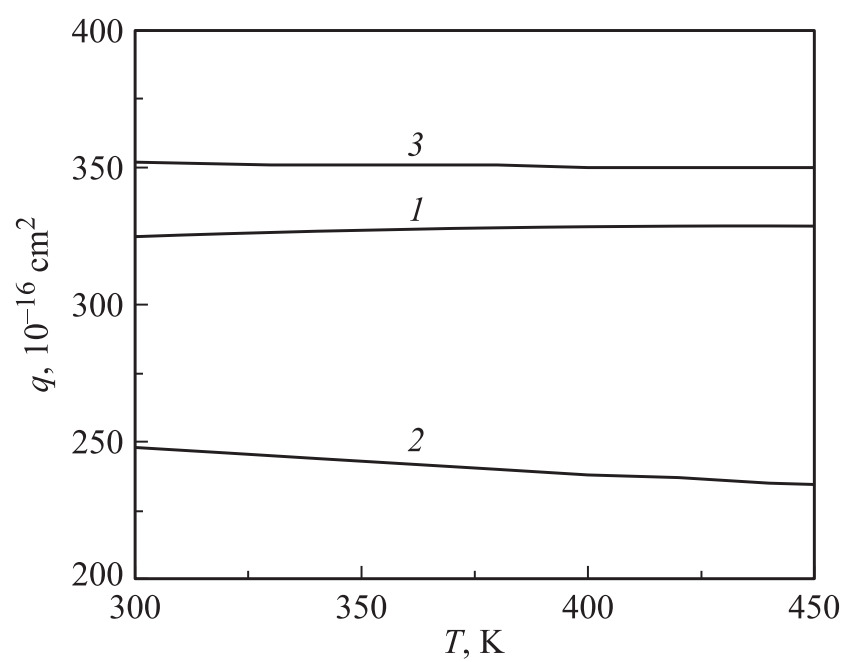

Рис. 1. Температурные зависимости действительных частей усредненных по скоростям комплексных сечений спинового обмена при столкновении атомов цезия и калия: $1-$ система $\mathrm{K}$-Cs [11], 2 - система $\mathrm{K}-\mathrm{K}$ [12], 3 - система Cs-Cs [10].

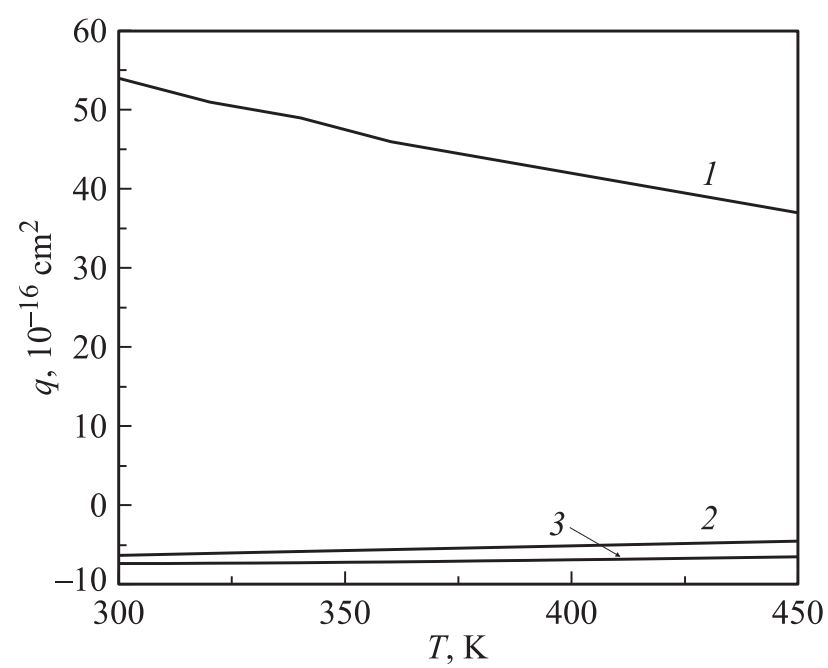

Рис. 2. Температурные зависимости мнимых частей усредненных по скоростям комплексных сечений спинового обмена при столкновении атомов цезия и калия: 1 - система $\mathrm{K}-\mathrm{K}$ [12], 2 - система Cs-Cs [10], 3 - система $\mathrm{K}-\mathrm{Cs}$ [11].

спинового обмена $\gamma$, зависящие от концентрации щелочных атомов, то необходимо учитывать, что в камере поглощения присутствует смесь щелочных атомов К и Cs. Концентрация атомов щелочных металлов определялась в соответствии с таблицами, приведенными в [22]. При использование смеси щелочных атомов для перехода от температуры камеры поглощения к концентрации в ней щелочных атомов необходимо воспользоваться законом Рауля для давления насыщенного пара над расплавом смеси металлов. В [8] предполагалось, что в расплаве присутствуют щелочные атомы двух сортов в равных весовых соотношениях. 

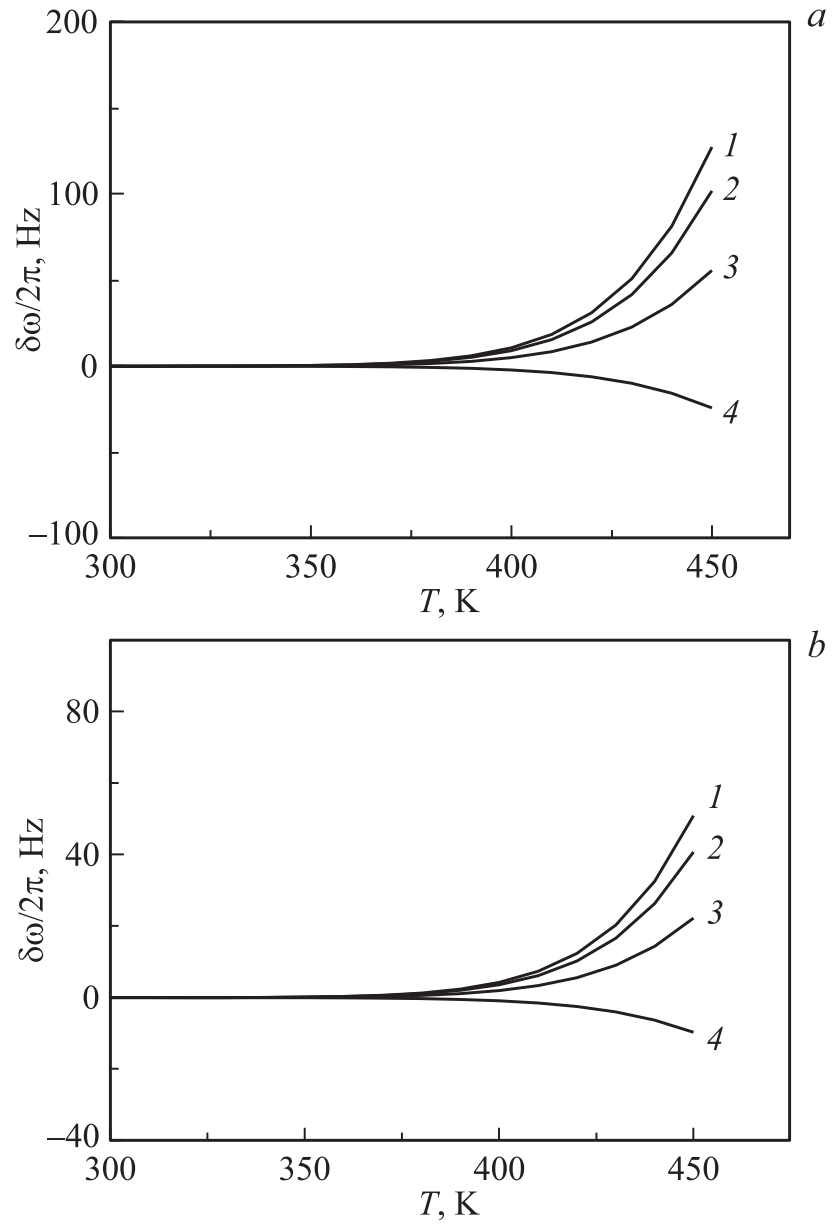

Pис. 3. (а) Зависимость сдвигов частоты магнитного резонанса от температуры при поляризации атома мишени в 50\% $\left(P_{z}(\mathrm{~B})\right): 1,2$ - атом мишени $\mathrm{Cs} ; 3,4-$ атом мишени $\mathrm{K} ; 1$ $\delta \omega(+)(\mathrm{K}-\mathrm{Cs}), 2-\delta \omega(-)(\mathrm{K}-\mathrm{Cs}), 3-\delta \omega(-)(\mathrm{Cs}-\mathrm{K}), 4-$ $\delta \omega(+)(\mathrm{Cs}-\mathrm{K}) . b)$ То же, что на $(a)$, при поляризации атома мишени в $20 \%$.

\section{3. Расчет спин-обменных сдвигов частоты для случая атомов $\mathrm{K}$ и Cs}

Расчет сдвигов частоты проводился на основании выражений (3)-(5) с учетом полученных ранее температурных зависимостей мнимых частей комплексных сечений спинового обмена. В таблице приведены значения коэффициентов из (3)-(7) для случаев, когда в качестве атома А выступает Cs или К. Результаты расчета приведены на рис. $3, a, b$. Как видно из рисунков, по мере увеличения температуры в камере поглощения (а следовательно, и концентрации щелочных атомов) сдвиги частоты как $\delta^{(1)} \omega(+)$, так и $\delta^{(1)} \omega(-)$ растут по абсолютной величине. При этом сдвиг $\delta^{(1)} \omega(+)$, обусловленный столкновениями атомов Cs c атомами K, остается отрицательным во всем диапазоне исследуемых температур, в то время как остальные сдвиги, а именно $\delta^{(1)} \omega(-)$ для пар $\mathrm{K}-\mathrm{Cs}$ и $\mathrm{Cs}-\mathrm{K}$ и $\delta^{(1)} \omega(+)$ для пары $\mathrm{K}-\mathrm{Cs}$ остаются положительными во всем диапазоне ис- следуемых температур. На рис. 3, $a, b$ приведены результаты расчетов для двух значений поляризации атомов В - 50 и $20 \%$.

Сдвиг частоты магнитного резонанса $\delta^{(2)} \omega$, представленный вторым членом в (2), существенно меньше, чем сдвиги $\delta^{(1)} \omega( \pm)$. Действительно, в магнитном поле порядка $10^{-4} \mathrm{~T}$ величина $\bar{\omega}_{0}=H_{0}\left|g_{s}\right| \mu_{B} / \hbar$ составляет порядка $10^{5} \mathrm{~Hz}$, величина в фигурных скобках выражения (5) при концентрации атомов порядка $10^{12} \mathrm{~cm}^{-3}$ составляет порядка $10^{6} \mathrm{~s}^{-1}$. С учетом величины $C$ из таблицы получается, что $\delta^{(2)} \omega / 2 \pi$ составляет порядка $10^{-4} \mathrm{~Hz}$, т.е. существенно меньше чем величины $\delta^{(1)} \omega( \pm)$, и сдвигами второго вида можно пренебречь.

\section{Конфликт интересов}

Автор заявляет, что у него нет конфликта интересов.

\section{Список литературы}

[1] Budker D., Romalis M. // Nature Physics. 2007. V. 3. N 4. P. 227-234.

[2] Александров Е.Б., Вершовский А.К. // УФН. 2009. Т. 179. № 6. C. 605-637.

[3] Groeger S., Bison G., Schenker J.L. et al. // Eur. Phys. J. D. 2006. V. 38. N 2. P. 239-247.

[4] Блинов Е.В., Житников Р.А., Кулешов П.П. // ЖТФ. 1979. T. 49. № 1. C. $588-596$.

[5] Gilles H., Hamel J., Cheron B. // Rev. Sci. Instr. 2001. V. 72. N 5. P. $2253-2260$.

[6] Картошкин В.А., Клементьев Г.В., Мельников В.Д. // ЖТФ. 1985. Т. 55. № 1. С. 131-136.

[7] Клементьев Г.В., Мельников В.Д., Картошкин В.А. // Химическая физика. 1985. Т. 4. № 1. С. 37-41.

[8] Александров Е.Б., Балабас М.В., Вершовский А.К., Пазгалев А.С. // ЖТФ. 2000. Т. 70. № 7. С. 118-124.

[9] Окуневич А.И. // Опт. и спектр. 1995. Т. 79. № 5. С. 718.

[10] Дмитриев С.П., Доватор Н.А., Картошкин В.А. // ЖТФ. 2015. T. 85. № 6. C. 40-43; Dmitriev S.P., Dovator N.A., Kartoshkin V.A. // Tech. Phys. 2015. V. 60. N 6. P. 826-829.

[11] Картошкин В.A. // Опт. и спектр. 2012. Т. 113. № 3. C. 264-268; Kartoshkin V.A. // Opt. Spectrosc. 2012. V. 113. N 3. P. 235-239.

[12] Картошкин В.A. // Опт. и спектр. 2011. Т. 111. № 6. C. 924-927; Kartoshkin V.A. // Opt. Spectrosc. 2011. V. 111. N 6. P. $881-884$.

[13] Дмитриев С.П., Доватор Н.А., Картошкин В.А., Окуневич А.И. // Опт. и спектр. 1994. Т. 77. № 5. C. 712-713; Dmitriev S.P., Dovator N.A., Kartoshkin V.A., Okunevich A.I. // Opt. Spectrosc. 1994. V. 77. N 5. P. 712.

[14] Картошкин В.A. // Опт. и спектр. 2010. Т. 108. № 6. C. 914-917; Kartoshkin V.A. // Opt. Spectrosc. 2010. V. 108. N 6. P. $866-869$.

[15] Xie F., Sovkov V.B. et al. // J. Chem. Phys. 2009. V. 130. P. 051102.

[16] Amiot C., Dulieu O. // J. Chem. Phys. 2002. V. 17. N 11. P. 5155-5164.

[17] Ferber R., Klincare I., Nikolayeva O. et al. // J. Chem. Phys. 2008. V. 128. P. 244316-1-9. 
[18] Ferber R., Klincare I., Nikolayeva O. et al. // Phys. Rev. A. 2009. V. 80. P. 062501-1-9.

[19] Amiot C. // J. Molec. Spectrosc. 1991. V. 146. N 2. P. 370-382.

[20] Amiot C., Verges J., Fellows C. // J. Chem. Phys. 1995. V. 103. N 9. P. 3350-3356.

[21] Ahmed E., Lyyra A.M., Xie F. et al. // J. Molec. Spectrosc. 2005. V. 234. N 1. P. 41-52.

[22] Несмеянов А.Н. Давление паров химических элементов. М.: Изд-во АН СССР, 1961. 396 с. 